\title{
Quand dire, c'est taire ? L' " effet de sourdine » racinien, stylistique et / ou rhétorique
}

\section{Anne Régent-Susini}

\section{(2) OpenEdition}

1 Journals

Édition électronique

URL : http://journals.openedition.org/rhetorique/95

DOI : $10.4000 /$ rhetorique. 95

ISSN : 2270-6909

Éditeur

UGA Éditions/Université Grenoble Alpes

Édition imprimée

ISBN : 978-2-84310-255-4

Référence électronique

Anne Régent-Susini, «Quand dire, c'est taire ? L'« effet de sourdine » racinien, stylistique et / ou rhétorique », Exercices de rhétorique [En ligne], 1 | 2013, mis en ligne le 12 novembre 2013, consulté le 10 décembre 2020. URL : http://journals.openedition.org/rhetorique/95 ; DOI : https://doi.org/ 10.4000/rhetorique.95

Ce document a été généré automatiquement le 10 décembre 2020.

\section{(c) (P)}

Les contenus de la revue Exercices de rhétorique sont mis à disposition selon les termes de la Licence Creative Commons Attribution - Pas d'Utilisation Commerciale - Partage dans les Mêmes Conditions 4.0 International. 


\title{
Quand dire, c'est taire? L'" effet de sourdine " racinien, stylistique et / ou rhétorique
}

\author{
Anne Régent-Susini
}

1 Chacun connaît les développements de Leo Spitzer sur l'effet de sourdine du «style classique» racinien («Die klassische Dämpfung in Racines Stil»), leur attrait, leur intérêt, leur fécondité (la notion d'effet de sourdine a été, depuis, appliquée à maints écrivains). Je souhaiterais m'appuyer sur cette brillante étude pour repenser, dans ce cadre précis, un partage disciplinaire particulièrement délicat dans la littérature d'Ancien Régime : celui qui sépare la stylistique et la rhétorique. Pour autant, il ne saurait s'agir de rejouer dans sa globalité le riche débat opposant ces deux approches. Ces questions, fondamentales, ont déjà donné lieu à d'importantes études, qui ont notamment rappelé les enjeux d'une réhabilitation de la rhétorique, ainsi que ses fondements historiques et culturels ${ }^{1}$. Ce n'est donc pas directement la légitimité de l'approche rhétorique en elle-même qui m'intéressera ici, pas plus que la concurrence qui pourrait s'établir entre elle et une approche stylistique. Le présent article adoptera une perspective délibérément plus restreinte, puisqu'il tentera de réinterroger, à partir des analyses spitzériennes, l'effet - ou les effets - produit(s) par la si singulière langue racinienne.

2 On sait en effet que selon Spitzer, l'écriture racinienne trouve son principe unificateur dans un "effet de sourdine» (Dämpfung). La métaphore musicale - ou plus exactement pianistique - qu'il emploie est loin d'être indifférente: envisager le théâtre racinien sous l'angle de l'« effet de sourdine ", c'est l'envisager comme une œuvre dont les effets sont produits par des variations d'intensité; en d'autres termes : c'est en proposer délibérément une lecture héritée du Sturm und Drang via le romantisme, et naturellement fort éloignée de la redécouverte du baroque qui, bien plus tard, permettra de remettre en lumière un autre type d'effet, fondé sur l'ornement, sur l'amplificatio et plus largement sur des variations codifiées. En ce sens, et nul ne s'en étonnera, l'approche de Spitzer (et la conception du classicisme français 
qu'elle reflète, "produit d'un apogé absolutiste, aristocratique et formel ${ }^{2}$ ») apparaît ancrée dans son siècle et dans sa culture - comme, du reste, les références critiques qu'il mobilise implicitement (Lessing, Schlegel ${ }^{3}$, Auerbach ${ }^{4}$ ) ou explicitement (Vossler ${ }^{5}$ ). Elle prend ainsi délibérément l'œuvre racinienne - et à travers elle le classicisme français - à rebours de ses propres catégories esthétiques, puisque Spitzer ne se prive pas de rappeler la «maxime » selon laquelle «Le français est un piano sans pédale ${ }^{6}$ ». Piano romantique contre clavecin classique, émotion contre raison, style contre rhétorique : les dichotomies qui sous-tendent le texte de Spitzer lui fournissent une armature théorique tout autant qu'une évidente portée polémique, au moment même où la jeune stylistique prend naissance sur les décombres de la rhétorique scolaire.

Car si la métaphore pianistique de la Dämpfung pourrait permettre d'envisager la diversité d'impressions produite par la "pédale d' $\operatorname{effet}^{7}$ ", et si Spitzer lui-même voit dans «l'alternance et l'imbrication» de la «formule» et du "chant lyrique » «le propre de Racine ${ }^{8} "$, c'est bien l'idée d'assourdissement ou d'atténuation qui constitue le fil directeur de son article, assourdissement considéré comme la marque, moins d'un auteur individuel que d'une certaine vision du monde, intellectualisante, distanciée, jouant, en somme, le logos contre le pathos ${ }^{9}$. Cependant, reformuler ainsi la thèse spitzérienne, c'est de toute évidence déjà la rhétoriciser. Or la rhétorique, si elle n'est pas totalement absente des pages fameuses des Études de style, y est pourtant fort discrète, au point qu'elle peut apparaître comme une sorte de point (délibérément) aveugle des analyses spitzériennes. Car en proposant de relire l'œuvre racinienne à la lumière d'un unique effet, l'effet de sourdine, Spitzer entend caractériser l'écriture racinienne per se, et à travers elle la Weltanschauung d'une époque et d'une culture ; autrement dit, il entend se placer au plan extra-scénique et s'attacher à la voix ${ }^{10}$ de Racine, représentant emblématique du "classicisme », non à celle de ses personnages, encore moins à celle de ses personnages engagés dans telle ou telle situation de parole.

Sans remettre en question ses analyses, je souhaiterais pour ma part réexaminer au plan intra-scénique les stylèmes renvoyant pour Spitzer à un effet de sourdine; j'essaierai de montrer que lorsqu'ils apparaissent, ils se trouvent justifiés, au plan intrascénique, par la stratégie rhétorique déployée par le personnage qui les emploie - sans que cela n'invalide, du reste, l'interprétation qu'en donne Spitzer au plan extrascénique.

\section{1.}

5 Un premier ensemble de stylèmes pointés par Spitzer paraît avant tout lié à l'inscription des énoncés dans un genre littéraire aussi codifié que la tragédie classique-même si cette détermination rhétorique n'exclut nullement qu'ils produisent un effet expressif spécifique. Ce sont essentiellement les marques du grand style, et en particulier d'un registre soutenu et ostensiblement littéraire. À l'orée de son article, Spitzer définit cette orientation stylistique comme en creux :

C'est un style sécularisé, formé à la conversation usuelle, qui parvient à sa hauteur et sa solennité essentiellement en renonçant au sensuel, au vulgaire et au pittoresque coloré ${ }^{11}$.

6 De cette « hauteur » relève par exemple le choix de mots « comme sein ou flanc au lieu de ventre", dans lesquels Spitzer voit, non seulement des «mot[s] noble[s]» (marqueurs diastratiques), mais encore des instruments de sourdine, dans la mesure où 
« le vague et le flou conviennent parfaitement à une poésie axée sur l'âme et l'idée ${ }^{12}$ »; y participent également des périphrases du type " porter ses pas » (au lieu de aller), que Spitzer, un peu plus loin, commente en ces termes: "l'action d'aller devient une marche majestueuse; plus elle est digne, moins elle est spontanée ${ }^{13}$.» En réalité, il semble que stylistique et rhétorique se combinent ici : si ces termes participent de ce que qu'on pourrait appeler une " dé-concrétisation » du référent, c'est aussi parce que ces marques d'un registre élevé, éloigné, par définition, du langage courant, relèvent du principe de l'aptum et des exigences génériques propres à un genre noble - qui n'est pas nécessairement, du reste, la tragédie classique. Étudiant les traductions en vers de l' Énéide, Françoise Berlan a en effet montré le lien qu'entretiennent, pour des raisons à la fois de registre et de rythme, les constructions prédicatives à verbe support (porter ses pas, faire une prière, etc.) non seulement avec l'alexandrin dramatique classique, mais encore avec son ancêtre renaissant ${ }^{14}$.

7 Certes, même si l'horizon d'attente du spectateur ou du lecteur d'une tragédie classique intègre ces exigences poétiques et rhétoriques, le fait qu'elles soient respectées n'en produit pas moins un effet expressif sensible - sans quoi, du reste, elles n'auraient aucune raison d'être. Pour autant, il est permis de se demander si l'effet produit, en l'occurrence, n'est pas d'éloignement plutôt que de sourdine : métaphore spatiale (du reste présente chez Spitzer) contre métaphore musicale, la substitution n'est pas indifférente, d'autant qu'elle permet de retrouver l'esthétique de la mise à distance thématisée dans la préface de Bajazet à partir d'une citation des Annales de Tacite :

On peut dire que le respect que l'on a pour les héros augmente à mesure qu'ils s'éloignent de nous: Major e longinquo reverentia. L'éloignement des pays répare en quelque sorte la trop grande proximité des temps. Car le peuple ne met guère de différence entre ce qui est, si j'ose ainsi parler, à mille ans de lui, et ce qui en est à mille lieues ${ }^{15}$.

Une telle citation a souvent été proposée comme justification théorique de l'effet de sourdine spitzérien ${ }^{16}$; pourtant, l'effet qu'elle revendique n'est pas simplement celui d'une atténuation ou d'une intellectualisation, mais bien plutôt, une sorte de mixte entre le pathos fascinant et distancié du suave mari magno et la captatio " pédagogique ", en quelque sorte, qu'évoque Pavel dans son Art de l'éloignement :

Il s'agit de faciliter l'accès à la vérité exemplaire exhibée par l'œuvre, de favoriser la démarche de l'attention en lui offrant des points d'appui lumineux et intelligemment choisis, et de recouvrir l'ensemble d'un vernis, d'un brillant, qui à la fois attire l'œil et le garde à distance ${ }^{17}$.

9 Autrement dit, les stylèmes relatifs aux normes qui régissent la tragédie classique ne sont certes pas privés d'efficacité expressive, et à ce titre sont tout à fait justifiables d'être intégrés dans une "étude de style »- mais ils peuvent également être compris dans une perspective rhétorique, au croisement d'une exigence de l'aptum, d'un pathos distancié mais non atténué, et de la matrice exemplaire (ou exemplifiante) qui soustend la tragédie.

10 Les autres stylèmes pointés par Spitzer peuvent être regroupés en faisceaux convergents :

- ceux qui mettent à distance l'individu (l'article indéfini au lieu du défini ${ }^{18}$, le pluriel au lieu du singulier, le nom propre au lieu de la première ou de la deuxième personne, etc.) ;

- ceux qui brouillent, floutent les contours, comme les relatives périphrastiques en ce que;

- ceux qui mettent à distance les faits en privilégiant l'intention sur l'action, ainsi les « verbes phraséologiques » tels que vouloir, oser, etc. ; 
- enfin, une dernière catégorie plus paradoxale regroupant des stylèmes qui pourraient fonctionner comme une Dämpferaufhebung, comme des instruments de dramatisation (ou d' amplification au sens rhétorique du terme), mais qui, selon Spitzer, contribuent au contraire chez Racine à instaurer une distance intellectualisante (le "démonstratif de distance », et surtout les intensifs si et tant et la personnification des abstraits).

11 Or il appert que dans la majorité de leurs emplois, ces stylèmes ne remplissent pas seulement une fonction poétique ou stylistique, mais également une fonction rhétorique : telle sera l'hypothèse principale de cet article, à l'appui de laquelle seront examinées quelques occurrences proposées par Spitzer dans son article ${ }^{19}$.

\section{2.}

Un premier ensemble d'exemples correspond aux antithèses, et en particulier aux oxymores, jugées par Spitzer trop intellectualisantes pour être passionnées :

L'oxymoron a quelque chose de trop élaboré, de trop mis en forme, il dénote trop d'ingéniosité pétrarquisante pour avoir une résonance affective. On peut en dire autant des antithèses, si fréquentes chez Racine, et qui ont le même rôle de fixation d'une antinomie ${ }^{20}$.

Spitzer illustre notamment cette remarque par une réplique prononcée par Oreste dans la scène II, 2 d'Andromaque (v. 537-538) : « [...]. Tel est mon partage funeste : /Le Cœur est pour Pyrrhus, et les vœux pour Oreste. » Il est indéniable que l'opposition binaire caractérise massivement ces deux vers, au plan stylistique (opposition entre les deux noms propres Pyrrhus et Oreste, opposition de sens et de nombre entre le singulier le cœur et le pluriel les vœux), comme aux plans syntaxique (hypozeuxe) et rythmique (césure fortement marquée permettant ainsi aux deux hémistiches de s'opposer nettement). Pour Spitzer, un tel système antithétique s'apparente à une virtuosité verbale renvoyant principalement à une vision intellectualisante, mais aussi descriptive, statique ("fixation des antinomies») et quelque peu schématique, de la situation $^{21}$, qui vaut antidote rationnel à la passion :

Dans la plupart des cas, il s'agit de révéler l'absurdité d'une passion ou d'un aveuglement [...]. Mais l'image antithétique ainsi donnée fait appel à l'intellect et à la raison, rétablit la suprématie de l'entendement ${ }^{22}$.

Il commente cet exemple en ces termes :

Un partage aussi tranché que celui du vers : Le cœur est pour Pyrrhus, et les vœux pour

Oreste, surprend, quand on songe à la complexité des opérations du cœur humain ${ }^{23}$.

Qu'une orchestration aussi systématique des antithèses puisse paraître au lecteur du $\mathrm{xx}^{\mathrm{e}}$ siècle trop construite pour être spontanée, trop rhétorique pour être naturelle semble compréhensible; de ce point de vue, la démarche herméneutique de Spitzer, foncièrement phénoménologique et ancrée hic et nunc, apparaît parfaitement cohérente - même si Gilles Declercq a montré combien les héros raciniens étaient à la fois passionnés et orateurs (fussent-ils orateurs malheureux). Cependant, il ne s'agit pas ici, du moins pour le personnage qui s'exprime sur scène, de « révéler l'absurdité d'une passion » ou de rendre compte des " opérations du cœur humain », mais d'agir sur son interlocuteur direct. La réplique d'Oreste ne peut en réalité se comprendre que comme une réponse à la réplique précédente, dans laquelle Hermione, humiliée par Pyrrhus, tentait de se rapprocher d'Oreste :

Vous que mille vertus me forçaient d'estimer ;

Vous que j'ai plaint, enfin que je voudrais aimer. [Andromaque, v. 535-536] 
Oreste, loin de décrire un état de fait, formule en réalité le présupposé contenu dans le verbe vouloir employé au conditionnel par Hermione ( je voudrais vous aimer » $=$ « je ne vous aime $\left.\operatorname{pas}^{24} »\right)$. Il exhibe également par cette antithèse la contradiction logique existant selon lui entre les verbes forcer et vouloir d'une part, estimer et aimer d'autre part. Autrement dit, il propose de l'amplification lénifiante d'Hermione un résumé polémique et cinglant, jouant sur la mise en œuvre brutale d'un argument proche de ce que Perelman appelle « la dissociation des notions ${ }^{25} »$. Plus exactement, il s'agit pour lui de faire éclater l'apparente unité du je d'Hermione, en suggérant l'inanité de la tension volontariste vers l'amour qu'elle programme ( je voudrais vous aimer $\left.{ }^{26} »\right)$, en refusant le présupposé que l'amour pourrait se déployer à partir d'un acte de la volonté. Il ne s'agit donc pas simplement d'opposer deux prédications (vous l'aimez/vous voudriez m'aimer) : la force argumentative d'une telle réplique tient au caractère "polaris[é] et inégalitaire » de la mise en opposition des notions considérées ${ }^{27}$ : de fait, «le cœur » et "les vœux» ne sont qu'en apparence placés sur le même plan; cette dichotomie se ramène peu ou prou à une dichotomie « le réel »/« l'illusion », tant le "Cœur » est tout ce qui importe à Oreste. Un tel vers n'a donc nullement vocation à décrire un état de fait, à ressaisir sous le sceau de la raison le clivage d'un de ces « personnages féminins, qu'on dit perpétuellement contradictoires ${ }^{28} "$. Chargé d'émotion, il vise à contraindre Hermione à sortir de son langage imprécis pour adopter une position plus franche, et équivaut, en réalité, à un double questionnement essentiel: "Oui ou non, m'aimezvous? ? et «Quid de mon rival Pyrrhus?». Si sophistication rhétorique il y a, elle réside moins dans l'orchestration formelle de l'antinomie que dans l'usage, à la manière d'une pointe, du mot polysémique vœux: employant le mot vœux au sens volontariste de promesse (c'est-à-dire du côté du vouloir et non du cœur) - sens auquel il croit, donc - , Oreste laisse néanmoins résonner, avec une ironie amère, le sens galant de ce vocable, très courant chez Racine et topique en contexte amoureux, mais auquel, en l'occurrence, il ne croit pas. Entrant en résonance, ces deux sens composent un effet de polyphonie remarquablement analysé par Gilles Siouffi à partir d'autres exemples raciniens ${ }^{29}$. Loin de produire un effet de sourdine, c'est bien un effet de radicalisation et d'entrechoquement, doublé d'un écho douloureux valant sarcasme, que produit la reformulation polémique proposée par Oreste.

D'autres exemples d'antithèses citées par Spitzer remplissent une fonction rhétorique encore plus évidente, ainsi la réplique d'Hermione à Oreste en IV, 3 (Andromaque, v. 1251-1252) : «Et tout Ingrat qu'il [Pyrrhus] est, il me sera plus doux / De mourir avec lui que de vivre avec vous. » Parler ici d'effet de sourdine, fût-ce pour désigner par là une expression par laquelle « le sentiment s'analyse lui-même au lieu de s'épancher uniment ${ }^{30}$ ", ne peut valoir qu'au niveau extra-scénique, dans la mesure où l'antithèse compose ici, au niveau intra-scénique, une proclamation d'une extrême violence rhétorique, violence absolument délibérée, dans un but précis : pousser Oreste à bout, exacerber sa souffrance, afin qu'il exécute publiquement la vengeance - ce qu'il fera, du reste. Là encore, il ne s'agit pas d'analyser ou de dépeindre un état de fait, mais bien d'agir sur l'autre pour le faire à son tour accomplir une action - et pas n'importe laquelle, puisqu'il s'agit du meurtre d'un souverain légitime.

17 Spitzer relève également, dans Phèdre, III, 1, l'antithèse sous-tendant la réplique d'CEnone (Phèdre, v. 754) : « Vous l'osâtes bannir, vous n'osez l'éviter. » De même que dans l'exemple précédent, le paradoxe ici exhibé par la structure binaire (dont la valeur adversative est d'autant plus évidente qu'elle demeure implicite, portée seulement par 
le quasi-hypozeuxe vous + oser + l(e ) + infinitif associée à l'opposition entre affirmation et négation ainsi que par une césure, là encore, très marquée) constitue non pas un constat, mais, de toute évidence, une mise en accusation de l'allocutaire prenant la forme d'un argument ad personam fondé sur le principe de contradiction, ou plus exactement sur un topos du type : «Il est préférable d'agir conformément à ses paroles que de démentir ses paroles par les actes ${ }^{31}$ ». De même, enfin, les oppositions sémantiques, rythmiques et syntaxiques structurant le vers d'Enone (Phèdre , v. 343-344 : «Sa mort vous laisse un Fils à qui vous vous devez/Esclave, s'il vous perd, et Roi, si vous vivez ») possèdent une évidente valeur argumentative, en soulignant l'alternative et en dramatisant par là même la portée délibérative d'une telle séquence.

Si, pour Spitzer, «le travail mental nécessaire à la dissociation de deux expressions soudées par Racine [...] vient ", dans tous ces passages, «troubler le plaisir poétique ${ }^{32}$ », c'est donc que sa conception de la poésie est en réalité, non seulement non rhétorique, mais anti-rhétorique, ou plus exactement peut-être, opposée à toute rhétorique qui se donnerait pour telle, qui ne se dissimulerait pas sous une apparence de naturel et de spontanéités ${ }^{3}$. En diachronie pourrait s'esquisser un lien avec le débat sur la possibilité de figures naturelles, dont on sait l'importance chez un Lamy, contemporain de Racine $^{34}$ - mais ce n'est naturellement pas l'objet de Spitzer, qui se positionne résolument en synchronie.

\section{3.}

Spitzer pointe également, comme en négatif, ce qu'il appelle le «remplissage de certains hémistiches avec des déterminations adverbiales, des appositions, des parenthèses ", qui produit selon lui à la fois une impression d'«atténuation » (de ouatage), de " mise en forme » (de formalisation quelque peu artificielle) - et, parfois, de précaution préparatoire avant une formule hardie, a priori difficile à accepter pour l'allocutaire. Il inclut dans ces procédés « de sourdine et de préparation » certaines "parenthèses" (incidentes), qu'il illustre notamment par l'exemple suivant, tiré de Phèdre (v, 1 v. 1345-1346) : «Je n'ai pu vous cacher, jugez si je vous aime, / Tout ce que je voulais me cacher à moi-même.» Or, l'incidente ne sépare pas seulement ici les deux membres de l'antithèse vous cacher/me cacher, laissant le premier en suspens (attente du COD) : elle fonctionne comme une communication, que Fontanier définit en ces termes : « Par la Communication, afin de mieux persuader ceux à qui ou contre qui l'on parle, [...] on a l'air de les consulter, d'entrer en conférence avec eux, et de s'en rapporter à ce qu'ils décideront eux-mêmes ${ }^{35}$ ». Là encore, il ne produit une impression de « remplissage » que si l'on considère qu'une telle figure rhétorique est devenue un artifice dépourvu de toute réelle efficacité. En choisissant de passer sous silence cette fonction rhétorique tout à fait repérable, c'est bien le procès d'une rhétorique devenue pur code institutionnel, écran artificiel à l'expression et à la contagion des passions tragiques, que semble dresser Spitzer.

Autre stylème relevé par Spitzer : « la répétition de mots ou de radicaux identiques ${ }^{36}$ ». Ce procédé topique est interprété, là encore, dans un sens rationalisant et associé, à ce titre, à l'effet de sourdine. Le premier exemple cité est le suivant, tiré de Phèdre, IV, 6, v. 1302 : « Mortelle subissez le sort d'une mortelle. » Or là encore, si l'antépiphore (répétition du même élément au début et à la fin de la structure syntaxique et métrique) «signifie une maîtrise de la situation ", c'est qu'elle renvoie à un argument 
de type logique sous-jacent. La quasi-tautologie apparente peut en effet être lue comme un avatar du lieu du genre ${ }^{37}$ qui, effectivement, opère une rationalisation du réel : il est rationnel, qu'une mortelle (Phèdre) subisse le sort réservé à son genre, puisse tomber sous le joug d'une passion illégitime. Cela est, en somme, conforme à la règle de justice - et, de fait, le lieu du genre fonctionne ici dans le cadre d'une rhétorique de l' excusatio, de la justification.

Les exemples pourraient ainsi être multipliés, qui attestent de la possibilité de compléter l'interprétation proposée par Spitzer en envisageant l'effet produit au niveau intra-scénique par les stylèmes qu'il met en lumière. En raison de son positionnement disciplinaire tout autant que de la relation polémique qu'il entretient avec la rhétorique traditionnelle, le critique autrichien choisit de minorer fortement la dimension argumentative des énoncés qu'il analyse, au profit d'une interprétation renvoyant soit à la psychologie du personnage, soit à la vision du monde propre à l'auteur ou à son époque. C'est ainsi qu'il passe délibérément sous silence la structure enthymématique d'un énoncé comme le célèbre : «Je t'aimais inconstant, qu'aurais-je fait fidèle?» (Andromaque, v. 1373), pour ne commenter que l'effet de condensation qu'elle produit, ramené à une sorte de traduction mimétique "d'une âme oppressée ». Le bénéfice argumentatif et rhétorique de l'ellipse de la majeure, de la question rhétorique, etc. n'est pas évoqué, puisque la réplique est donnée comme une émanation directe et transparente de l'« âme » du personnage. De manière analogue, évoquant les «invocations à des puissances surnaturelles » ou plus largement à des instances inanimées, telles « des villes ou autres lieux ${ }^{38}$ », Spitzer y voit du " pathétique stylisé » et considère que Racine « intériorise » ce procédé, qui trouve son origine dans le lien entre la tragédie antique et le cérémonial religieux dans lequel elle s'inscrivait :

Dans l'antiquité, où la tragédie procède directement des rites religieux, il était compréhensible que le personnage théâtral tourne ses regards vers la divinité proche; pour un auteur chrétien, dont le Dieu ne se trouve nulle part, et surtout pas à proximité des bancs « où sont assis les moqueurs", ces apostrophes sont des éléments formels ${ }^{39}$.

Pour être topique, la question, du reste, n'en revêt pas moins d'importance : sortie de son contexte antique, la rhétorique est-elle devenue un code "refroidissant ${ }^{40} »$ ? Sa puissance et son dynamisme se sont-ils émoussés au point de transformer la symphonie des figures en une unique sourdine? Quoi qu'il en soit, dans le cas de l'apostrophe oratoire, il semble bien que pour Spitzer, le procédé rhétorique, privé de sa justification profonde, soit devenu un moyen au service du pathétique, certes, mais d'un pathétique purement expressif (et non agissant), tourné vers soi-même, l'expression intériorisée d'un trouble :

Le personnage s'adresse à sa propre âme, à ses propres sentiments; ainsi, le regard théoriquement tourné vers le haut s'abaisse dans les profondeurs de l'intériorité des personnages, véritable séjour du dieu invoquét1.

L'un des exemples cités est celui d'Andromaque s'adressant à Pyrrhus en III, 6 : J'ai cru que sa Prison deviendrait son asile. Jadis Priam soumis fut respecté d'Achille. J'attendais de son Fils encor plus de bonté. Pardonne, cher Hector, à ma crédulité. Je n'ai pu soupçonner ton ennemi d'un crime ; Malgré lui-même enfin je l'ai cru magnanime. Ah! s'il l'était assez pour nous laisser du moins $\mathrm{Au}$ Tombeau qu'à ta Cendre ont élevé mes soins, 
Et que, finissant là sa haine et nos misères,

Il ne séparât point des dépouilles si chères ! [Andromaque, v. 941-959]

Or l'apostrophe oratoire "cher Hector", ici, ne dit pas seulement la souffrance d'Andromaque pleurant son époux, pas plus qu'elle ne s'adresse à "la propre âme " d'Andromaque; tournée vers l'interlocuteur bien réel de la veuve, Pyrrhus, elle lui oppose la grande figure du héros défunt (après lui avoir opposé celles de Priam et d'Achille ${ }^{42}$ ). Il s'agit bien d'une apo-strophe, consistant à se tourner (en apparence) vers un autre que le juge pour mieux influencer ce dernier ${ }^{43}$. Une telle figure permet en outre de formuler indirectement, à la troisième personne, la requête provocatrice adressée à Pyrrhus (« laissez-nous mourir !») - qu'il ne pourra, du reste, que refuser. Pas plus que l'hypotypose dans d'autres contextes ${ }^{44}$, l'apostrophe oratoire n'a pour fonction d'exprimer simplement la passion qui possède le personnage racinien.

Tous ces procédés revêtent donc, dans la plupart de leurs emplois, une fonction argumentative essentielle, et soulignent combien chez Racine, passion et rhétorique sont loin de s'opposer : très rarement réduit au silence ou à la confusion d'une parole anarchique, le personnage passionné produit généralement un discours certes parfois sophistique, mais sophistiqué : sa parole n'est pas incohérence, mais, comme l'écrit Gilles Declercq, "cohérence téléologiquement égarée 45 ». L'approche proposée par Spitzer regroupe ainsi sous la dénomination d'effet de sourdine des procédés qui, audelà de leur diversité formelle, présentent une diversité de fonctionnements rhétoriques tout à fait remarquable.

\section{4.}

Cette dimension rhétorique n'est du reste pas totalement absente des Études de style, où elle apparaît par moments, fugitivement et comme en creux, à l'occasion de certaines analyses. C'est ainsi que Spitzer mentionne l'atténuation de l'audace que peut permettre l'effet de sourdine ${ }^{46}$; il souligne de même, s'agissant de la scène IV, 4 de Mithridate, que la froideur qu'y manifeste Monime possède un fondement rhétorique précis, puisqu'il s'agit, précisément, de «repousser Mithridate ${ }^{47}$ »; il remarque enfin que les verbes de modalité vouloir, oser, daigner, etc. "magnifie[nt] $1[\mathrm{e}]$ discernement " des personnages qui les emploient, "leur responsabilité, la souveraineté de leurs résolutions » - c'est-à-dire participent à la construction d'un ethos héroïque.

Par ailleurs, les valeurs stylistique et rhétorique d'un même trait formel peuvent parfois se superposer, comme dans le cas des stylèmes marquant une tension vers le général. Car outre la méfiance envers l'individuel et l'attraction vers la règle souvent associées au classicisme, outre la minoration d'un pathos d'autant plus abstrait qu'il se trouve ramené à des schèmes donnés pour universels, la prégnance des stylèmes généralisants peut également s'expliquer, en bien des cas, par des considérations rhétoriques, dans le cadre, par exemple, d'une stratégie de l'excusatio articulée à une anthropologie et à une théologie mettant l'accent sur l'impuissance de l'homme déchu (si j'agis ainsi, c'est que je représente - malgré moi - tel type, c'est que j'obéis - malgré moi - à telle loi générale, etc.). En témoigne, parmi bien d'autres exemples, telle réplique d'Oreste dans Andromaque, III, 1 :

J'abuse, cher ami, de ton trop d'amitié.

Mais pardonne à des maux dont toi seul as pitié ; 
Excuse un malheureux qui perd tout ce qu'il aime,

Que tout le monde hait, et qui se hait lui-même. [Andromaque, v. 799-802] fondamentalement distincte d'une approche rhétorique, à deux titres au moins. D'une part en raison de la méthode employée, à savoir celle de la microlecture, de l'analyse d'exemples isolés - alors que la lecture rhétorique (si l'on entend la rhétorique en un sens large, et non au sens de rhétorique des figures) suppose de prendre en compte des masses signifiantes plus étendues, qu'il s'agisse de la période ou de la tirade. D'autre part en raison du niveau (au sens énonciatif du terme) auquel se place l'analyste. Le théâtre, comme on sait, est le lieu par excellence de la "double énonciation »; or, Spitzer, se situant au plan de la réception, de l'effet produit sur «le lecteur » ou «l'auditeur » (car chez lui il n'est jamais question de spectateur, pas plus, du reste, que d'acteur), s'en tient résolument au niveau extra-scénique: c'est Racine qui parle, qui nous parle. De ce point de vue, Spitzer n'est pas si éloigné des tenants de la "poésie pure » chez Racine et de l'archi-personnage ou du récitant lyrique unique qu'elle suppose $^{48}$.

Ces deux lieux de divergence s'avèrent du reste fondamentalement liés : c'est parce qu'il procède essentiellement par microlectures que chez Spitzer, le contexte n'est presque jamais donné, et c'est parce que ce contexte n'est presque jamais donné que la voix de tous les personnages semble équivaloir à une voix unique, celle de Racine. Il peut sembler symptomatique, à cet égard, que Spitzer, dans un article célèbre, se soit tout particulièrement intéressé au récit de Théramène, celui-là même qui était critiqué par Houdar de La Motte comme l'exemple-type de la description « où l'on croit plutôt entendre l'auteur que le personnage qu'il fait parler ${ }^{49}{ }$ - l'évocation étant jugée trop sophistiquée pour pouvoir vraisemblablement être attribuée à Théramène épouvanté. Sans doute n'est-il pas indifférent, en effet, que ce soit à ce passage, le moins « récupérable " peut-être au niveau intra-scénique (en dépit de la position implicite d'accusé qu'occupe à ce moment Thésée), que le critique autrichien ait consacré un article spécifique - article, du reste, et non sans paradoxe, beaucoup plus accueillant à la perspective rhétorique que celui des Études de style. C'est bien entre Racine et «le lecteur» ou "l'auditeur» que se joue principalement, voire exclusivement, pour Spitzer, ce qui intéresse l'étude de style.

Or, sans remettre en cause la fécondité de ses analyses, il paraît possible d'articuler autour de l'« effet de sourdine » les deux niveaux de la double énonciation théâtrale. La fascination que peut exercer le théâtre de Racine pourrait en effet tenir à la conjonction de deux caractéristiques formelles.

D'une part, une identité stylistique propre, indéniable, aisément identifiable, qui transcende la diversité des situations, des entreprises de persuasion représentées sur scène, et par laquelle l'œuvre unifiée qu'est le théâtre de Racine, en proposant une certaine manière de représenter le monde, incarne et accomplit tout à la fois un certain idéal esthétique de son temps. À cet égard, le repérage précis qu'effectue Spitzer, à l'échelle de l'œuvre tout entière, d'un ensemble de procédés renvoyant à un effet global d'expression maîtrisée des pulsions passionnelles, est aussi décisif qu'éclairant - ce dont témoigne du reste la place occupée par ses analyses dans le panthéon de la critique racinienne. Tout au plus pourrait-on juger préférable d'inscrire ces procédés, plutôt que dans le cadre d'un effet de sourdine, dans le cadre d'une esthétique alliant, d'une manière qui lui est propre, l'éloignement, en lien avec une stylistique des genres 
(c'est la distance propre à la tragédie) et, comme l'a suggéré Gilles Declercq, la sobriété, en lien avec une stylistique de groupe, en l'occurrence l'« esthétique sévère » de PortRoyal ${ }^{50}$. Non seulement, en effet, la sobriété ne renvoie pas nécessairement à la diminution d'intensité que semble impliquer en français la métaphore de la sourdine ; mais elle semble en outre une voie plus naturelle vers le sublime qu'une sourdine quelque peu affadissante; envisager l'effet de sourdine en termes de sobriété permettrait en dernière instance de rendre compte de la singularité paradoxale du style racinien, entant les charmes d'une galanterie souvent considérée comme féminine sur l'atticisme viril et grave d'un Champaigne.

31 D'autre part, la mobilisation constante d'une rhétorique sophistiquée - qui, dans la plupart des cas, vient motiver, au plan intra-scénique, les stylèmes désignés par Spitzer comme emblématiques du classicisme, leur conférant dès lors une double résonance, stylistique (ou esthétique) et rhétorique.

Or il est permis de penser que même du point de vue de la réception, auquel se place Spitzer, ces deux niveaux sont actualisés, dans la mesure où le spectateur perçoit à la fois l'intérêt stratégique, pour le personnage qui les utilise, de ces formulations distanciées, et leur beauté poétique, celle-ci pouvant naître tout autant du contraste entre violence des passions et maîtrise de l'expression, que du spectacle tragique d'une belle rhétorique déployée en vain ${ }^{51}$.

Reste que pour percevoir pleinement ces deux niveaux, et donc pour goûter l'effet de sourdine dans toute sa richesse, le spectateur/lecteur doit posséder une certaine maîtrise de l'héritage rhétorique que mobilise Racine. Or au moment où écrit Spitzer, cet héritage joue dans la réception du texte racinien un rôle à la fois de plus en plus restreint et de plus en plus problématique, la stylistique naissante se construisant à bien des égards contre la rhétorique scolaire. Dès lors, on peut comprendre que, prenant acte d'un recul de fait tout autant que d'un reniement polémique de cet héritage, les analyses spitzériennes ne puissent qu'être encadrées par le constat mélancolique que l'œuvre de Racine ne touche plus la plupart des lecteurs, à l'étranger comme en France :

Le lecteur moderne (surtout s'il est allemand) est presque toujours déconcerté par le style racinien [...]. La sourdine mise par Racine dans son style lui ferme le cœur des Français d'aujourd'hui ${ }^{52}$.

De fait, passer sous silence le niveau intrascénique de l'analyse, laisser de côté le dynamisme persuasif des échanges entre les personnages, c'est peut-être prendre le risque de ne voir dans l'effet de sourdine qu'un effet "refroidissant». Car le niveau intrascénique est au contraire celui où règne ce qu'Emmanuel Bury appelle un " réalisme argumentatif [...] qui conduit à une homogénéité du signe théâtral et de son public ", au sens où, d'après Quintilien, il n'y a pas de différence fondamentale (et donc pas de différence au plan perlocutoire) entre une délibération simulée et une délibération réelle ${ }^{53}$, ce qui permet, au moment où se déploie une entreprise rhétorique sur scène, une forme de fusion entre public et acteurs, tous impliqués et convoqués par le discours qui leur est donné à entendre.

Stylistique et rhétorique, donc : prendre en compte les deux strates d'énonciation de la parole théâtrale, dont l'une (de l'auteur au spectateur/lecteur) peut être envisagée en termes d'éloignement tandis que l'autre (de personnage à personnage) est le lieu d'une " contagion d'un pathétique maîtrisé grâce à la connaissance intime de l'art oratoire ", dans le cadre d'une « esthétique de la fusion et de la participation ${ }^{54}$ », permet peut-être 
au contraire de restituer la manière paradoxale qu'a l'œuvre racinienne de nous toucher.

\section{NOTES}

1. Voir notamment G. Declercq, «Poéticité versus rhétoricité » dans R. W. Tobin éd., Racine et / ou le classicisme, Tübingen, Gunter Narr, 2001, p. 20, n. $4:$ : la littérature classique appartient à ce que Marc Fumaroli a nommé "l'âge de l'éloquence", c'est-à-dire la période où la rhétorique [a possédé] une double fonction matricielle (elle forme les écrivains) et formelle (elle prête ses structures aux œuvres littéraires). De surcroît, dans le cas de la littérature dramatique où les personnages sont orateurs, la structure textuelle est directement déterminée par le tissage rhétorique du discours. Cette rhétoricité, enfin, conditionne la déclamation de l'acteur tragique au XVII ${ }^{\mathrm{e}}$ siècle. »

2. D. Meur, «Introduction » dans E. Auerbach, Le Culte des passions. Essais sur le XVII ${ }^{e}$ siècle français, Paris, Macula, 1998, p. 24. Voir aussi ibid. : "C'est par référence à cette vision romantique du classicisme que se constituent durablement les clichés nationaux qui, de part et d'autre du Rhin, auront une fortune bien plus que littéraire : aux Français la belle et froide clarté classique, la rationalité cartésienne, l'élégance d'un esprit épuré de tout particularisme; aux Allemands la profondeur d'âme, l'abstruse spéculation, le goût du naïf, du local et de l'héroïque ».

3. Sur la réception de Racine en Allemagne, voir notamment E. Köhler, «Racine-Rezeption in Deutschland" dans H. Krauss et D. Riegerhrsg éd., Vorlesungen zur Geschichte der französischen Literatur, t. 3/1 : Klassik, Stuttgart/Berlin/Köln, Kohlhammer, 1983. Je remercie J.-Y. Vialleton pour la discussion éclairante que nous avons eue sur ces questions.

4. Voir notamment E. Auerbach, «Racine et les passions» (Le Culte des passions, op. cit.), qui s'inscrit explicitement dans la lignée de l'ouvrage alors récent de G. Truc (Jean Racine, Paris, Bibliothèque d'Histoire littéraire et de critique, 1926) et en opposition - quoique modérée - à Vossler. Sur l'influence qu'exerçait alors Vossler sur la romanistique allemande (et, par ailleurs, sur la manière dont y prédominaient alors les études dix-septiémistes), voir A. Varvaro, « Mimésis avant Mimésis » dans P. Tortonese éd., Erich Auerbach. La littérature en perspective, Paris, Presses de la Sorbonne nouvelle, 2009, p.73-74. Sur le champ épistémologique de la romanistique allemande, dans lequel s'inscrit Spitzer - comme Auerbach, Curtius et Vossler -, voir D. Combe, «Genres et styles chez Erich Auerbach », ibid., p. 192-193.

5. C'est également par rapport à Vossler que se positionne Spitzer au début de son article en français - le seul - sur « Racine et Goethe » (Revue d'histoire de la philosophie et d'histoire générale de la civilisation, janvier 1933, p. 58-75), p.60. Sur l'«école allemande de stylistique ", et en particulier sur Vossler et Spitzer, voir E. Karabetian, Histoire des stylistiques, Paris, Armand Colin, 2000, chap. 4, p. 70 sq.

6. L. Spitzer, "L'effet de sourdine dans le style classique:Racine» dans Études de style, trad. É. Kaufholz, A. Coulon, M. Foucault, Paris, Gallimard, 1996 (1980), p. 209.

7. En effet, la même pédale qui permet la Dämpfung permet aussi la Dämpferaufhebung, c'est-à-dire la levée de la sourdine - avec les effets de contraste (et éventuellement de paradoxe, l'intensité de l'émotion pouvant être soulignée par la sourdine qui aurait dû au contraire la voiler) qui en résultent. 
8. L. Spitzer, ibid., p. 208. Pour une relecture de ces analyses en lien avec la notion de sublime, voir G. Declercq, «Représenter la passion : la sobriété racinienne ", Littératures classiques, $\mathrm{n}^{\circ} 11$ (1989), p. 87-88. Dans son «Racine et Goethe» (op. cit., p. 73), Spitzer met en relation cette dualité de Racine avec une esthétique du montrer/cacher et de l'insinuation: "On a remarqué combien est essentiel pour l'art verbal de Racine le fait de cacher et de montrer en même temps ce qu'il aime à dépeindre, les passions : on trouve partout dans Racine des passages qui parlent de la difficulté de les traduire en langage humain et les grands effets de Racine sont précisément les moments où sa langue à lui rompt les digues de ses alexandrins et où la passion éclate à travers cette région moyenne qu'est le langage traditionnel de la scène française. »

9. Voir L. Spitzer, «L'effet de sourdine dans le style classique : Racine», op. cit., p. 209 : «Si j’ai associé, dans mon titre, le mot passe-partout de "classique" au terme de "sourdine", c'est parce que c'est précisément cet effet de sourdine qui crée dans le style de Racine l'impression de retenue et d'équanimité, que l'histoire littéraire attache à l'idée de classicisme ».

10. C'est le terme de "voix» qu'emploie Spitzer dans "Racine et Goethe », op. cit., p. 73 : "Racine, le grand poète de la tradition classique, qui à travers une langue traditionnelle et un répertoire poétique et moral hérité a su faire entendre jusqu'à nous sa voix personnelle. »

11. Op. cit., p. 208. Sur la notion de sécularisation appliquée au classicisme français chez Auerbach, voir Diane Meur, op. cit., p. 28.

12. Op cit., p. 233.

13. Ibid., p. 243.

14. F. Berlan, « Avènement du nom abstrait dans les traductions en vers du chant IV de l'Énéide de Du Bellay à Scudéry » dans F. Berlan et G. Siouffi dir., Langue littéraire et changements linguistiques, Paris, Presses d'Université Paris-Sorbonne, 2006, p. 240 : «Ces syntagmes binaires associent une plus grande efficacité sonore à un effet de registre donné par surcroît. » F. Berlan en décèle la présence jusqu'à notre époque, dans « un oral sinon soutenu, du moins officiel et à réception collective dont le type est constitué par l'information radiophonique ou télévisuelle. »

15. Racine, Bajazet, «Préface » (1676-1697) dans Euvres complètes I, éd. G. Forestier, Paris, Gallimard, 1999, p. 625.

16. Voir par exemple M. Prigent, J.-C. Darmon et M. Delon dir., Histoire de la France littéraire. Classicismes, Paris, Presses Universitaires de France, 2006, p. 15.

17. T. Pavel, L'Art de l'éloignement. Essai sur l'imagination classique, Paris, Gallimard, 1996, p. 54.

18. Pour Spitzer, l'écart stylistique relève moins d'une option expressive individuelle qu'il ne reflète une évolution collective, celle d'une langue et, tout à la fois, du peuple qui la parle. Comme la notion d'écart, l'imaginaire linguistique sous-tendant plus généralement une telle approche est caractéristique d'un certain moment de la réflexion menée sur l'histoire de la langue et des formes.

19. La plupart de ces exemples seront empruntés à Andromaque et à Phèdre, car ce sont les pièces les plus fréquemment citées par Spitzer - avec Bajazet et, dans une moindre mesure, Athalie - sans doute parce que ce sont les tragédies qui, dans la tradition de la romanistique allemande du XIX ${ }^{\mathrm{e}}$ siècle, sont les plus représentatives de l'œuvre de Racine.

20. L. Spitzer, op. cit., p. 257.

21. Voir aussi ce que dit Spitzer des "antithèses sanglantes" de Racine dans son "Racine et Goethe », op. cit., p. 64 : « Racine laisse subsister dans toute sa cruauté l'état du pécheur, entre le ciel et la terre, avec ses aspirations supraterrestres et avec ses faiblesses humaines, l'antithèse entre l'homme et Dieu (Phèdre et Dieu, par exemple). "

22. L. Spitzer, «L'effet de sourdine dans le style classique : Racine », op. cit., p. 259.

23. Ibid., p. 259.

24. Oreste répétera un peu plus loin (Andromaque, v. 542) : «Vous me voulez aimer et je ne puis vous plaire. "

25. C. Perelman, L’Empire rhétorique. Rhétorique et argumentation, Paris, Vrin, 1997, p. 139. 
26. Voir ce qu'elle a dit plus haut à Cléone (Andromaque, v. 474) : "Et peut-être il saura se faire aimer lui-même. "

27. G. Declercq, L'Art d'argumenter. Structures rhétoriques et littéraires, Paris, Éditions universitaires, 1992, p. 135.

28. L. Spitzer, op. cit., p. 259.

29. G. Siouffi, «Racine au risque de la clarté française » dans D. Denis dir., L'Obscurité. Langage et herméneutique sous l'Ancien Régime, Bruxelles, Academia Bruylant, 2007, p. 242-245.

30. L. Spitzer, op. cit., p. 259.

31. E. Eggs, Grammaire du discours argumentatif, Paris, Kimé, 1994, p. 93. Voir également C. Plantin, Essais sur l'argumentation, Paris, Kimé, 1990, p. 209.

32. L. Spitzer, op. cit., p. 262.

33. En cela, l'approche de Spitzer dans «L'effet de sourdine dans le style classique : Racine » nous semble se démarquer nettement de celle d'un Auerbach, et plus largement de la tradition philologique et académique allemande, qui continue à faire place à la rhétorique. Voir D. Combe, "Genres et styles chez Erich Auerbach », op. cit., p. 196-197.

34. Voir aussi, à propos des oxymores du type « heureuse cruauté » (Andromaque, II, 5), L. Spitzer, op. cit., p. 254 : «Si le jugement contenu dans les épithètes appréciatives détruit la spontanéité lyrique de l'épanchement des sentiments, cette froideur moraliste se renforce particulièrement lorsque le jugement contenu dans l'épithète est inattendu, parce que normalement incompatible avec le substantif en question: c'est le cas, fréquent chez Racine, de l'oxymoron, qui produit l'effet de surprise d'une pointe et montre au moins un recul du locuteur par rapport à ses propos. Cet esprit, cette lucidité, ne se retrouvent pas chez une personne emportée par la passion. »

35. P. Fontanier, Les Figures $d u$ discours, Paris, Champs Flammarion, 1977, article «Communication », p. 414.

36. Plus largement, pour une analyse poétique des phénomènes de répétition lexicale chez Racine, non plus en termes d' « effet de sourdine ", mais en termes d'« effet de résonance », voir B. Louvat, «Le vocabulaire à l'épreuve de la langue : l'exemple d'Andromaque », Revue La Licorne, Numéro 50, URL : http://licorne.edel.univ-poitiers.fr/document4394.php

37. Sur la notion de genre telle qu'elle est alors utilisée en logique et en rhétorique, voir R. Bary, La Rhétorique Française, où pour principale argumentation on trouve les secrets de notre langue, Paris, Le Petit, $1^{\text {re }}$ éd. 1653, 1665, p. 76. Sur les «lieux communs du genre », voir tout le livre IV des Topiques d'Aristote ainsi que Cicéron, Topiques, II, 13.

38. L. Spitzer, op. cit., p. 295.

39. Ibid., p. 295.

40. Voir par exemple ibid., p. 279 : «On trouve certes chez Racine mainte tirade exaltée, où les sentiments du personnage semblent s'épancher sans entrave, et pourtant en y regardant de plus près, on discerne le recours continuel à certains procédés techniques déterminés pour dépeindre l'égarement ; ce sont des procédés rhétoriques hérités des anciens, et dont l'emploi systématique formalise et stylise le discours ; c'est un égarement qui n'oublie pas d'ajuster artistement les plis de l'éloquence!"

41. Ibid., p. 279.

42. Voir M. Hawcroft, "L'apostrophe racinienne" dans G. Declercq et M. Rosellini dir., Jean Racine, 1699-1999, Paris, Presses Universitaires de France, 2003, p. 411 : « Si nous voulons bien comprendre comment les tragédies de Racine nous affectent en tant que spectateurs, nous devons nous rappeler que, du moins pour les scènes dialoguées, nous sommes les observateurs privilégiés de confrontations extrêmement conflictuelles entre des personnages s'acharnant à rallier les uns et les autres à leur propre point de vue. Alors si un personnage a recours à une technique rhétorique, ce n'est pas en premier lieu pour nous les spectateurs, c'est pour le personnage qui l'écoute. Et si une apostrophe exprime les émotions du locuteur, nous, en tant que spectateurs, devons y réagir en considérant l'effet produit sur l'autre personnage sur scène. » 
43. Voir Quintilien, Institution oratoire, IV , 1, 63.

44. Voir G. Declercq, «Une voix doxale : l'opinion publique dans les tragédies de Racine », XVII Siècle, vol. 46, nº 182 (1994), p. 117-118.

45. G. Declercq, « Poéticité versus rhétoricité », op. cit., p. 49, n. 48.

46. L. Spitzer, op. cit., p. 212.

47. Ibid., p. 240.

48. Voir G. Declercq, « Poéticité versus rhétoricité », op. cit., p. 31.

49. Houdar de La Motte, Discours sur la poésie en général et sur l'ode en particulier, Paris, Grégoire Dupuis, 1709, p. 37

50. Voir G. Declercq, "Stylistique et rhétorique au $\mathrm{XVII}^{\mathrm{e}}$ siècle: l'analyse du texte littéraire classique ", XVII Siècle, 152 (juillet-août 1986), p. 219 ; et id., "Représenter la passion : la sobriété racinienne ", op. cit., en particulier p. 87 sq.

51. Voir G. Declercq, « Poéticité versus rhétoricité », op. cit., p. 49.

52. L. Spitzer, op. cit., p. 208-314.

53. Voir E. Bury, « Mémoire, doxa et argumentation : le délibératif à l'œuvre dans la dramaturgie racinienne », dans Jean Racine, 1699-1999, op. cit., p. 393.

54. Ibid.

\section{AUTEUR}

\section{ANNE RÉGENT-SUSINI}

Université Sorbonne nouvelle - Paris III 